\title{
miR-16 inhibits hyperoxia-induced cell apoptosis in human alveolar epithelial cells
}

\author{
ZHIXI LI ${ }^{1}$, WENJUN JIANG ${ }^{1}$, GANG WU ${ }^{2}$, XUEMING JU ${ }^{3}$, YOUYU WANG ${ }^{4}$ and WENYING LIU ${ }^{1}$ \\ Departments of ${ }^{1}$ Pediatric Surgery, ${ }^{2}$ Hepatobiliary Surgery, ${ }^{3}$ Ultrasound and ${ }^{4}$ Thoracic Surgery, Hospital of The University of \\ Electronic Science and Technology of China and Sichuan Provincial People's Hospital, Chengdu, Sichuan 610072, P.R. China
}

Received August 2, 2017; Accepted November 13, 2017

DOI: $10.3892 / \mathrm{mmr} .2018 .8636$

\begin{abstract}
The identification and development of novel therapeutic strategies for acute lung injury is urgently required. It has been previously demonstrated that microRNA (miR)-16 suppresses the level of transforming growth factor (TGF)- $\beta$ in acute lung injury (ALI). Therefore, the present study investigated the role of miR-16 in the phenotype, cell proliferation and apoptosis, and the involvement of TGF- $\beta /$ Smad family member $2(\operatorname{Smad} 2)$ and JAK/signal transducer and activator of transcription (STAT)3 signaling, of primary human alveolar type II epithelial cells (AECII). Following transfection with miR-16 mimics, AECII cells were exposed to hyperoxia for $24 \mathrm{~h}$. Subsequently, immunofluorescence staining of surfactant protein-A (SP-A) was performed, and cell proliferation and apoptosis were investigated by Cell Counting Kit- 8 assays and annexin V-fluorescein isothiocyanate/propidium iodide staining, respectively. Furthermore, the expression levels of miR-16, TGF- $\beta$, Smad2, phosphorylated-Smad2, JAK and STAT3 were detected by western blotting and/or reverse transcription-quantitative polymerase chain reaction. The results demonstrated that miR-16 levels and SP-A fluorescence were markedly inhibited by hyperoxia. Furthermore, transfection of AECII cells with miR-16 mimics increased SP-A fluorescence in hyperoxia-treated AECII cells, significantly reversed hyperoxia-induced reductions in cell proliferation and inhibited hyperoxia-induced apoptosis. Finally, miR-16 mimics modulated the mRNA and protein expression of components of the TGF- $\beta /$ Smad 2 and JAK/STAT3 pathways in AECII cells following hyperoxia. In conclusion, the results of the present study indicate that overexpression of miR-16 may exert a protective effect in AECII cells against cell apoptosis and ALI, which may be associated with TGF- $\beta /$ Smad2
\end{abstract}

Correspondence to: Dr Wenying Liu, Department of Pediatric Surgery, Hospital of The University of Electronic Science and Technology of China and Sichuan Provincial People's Hospital, 32 Yihuan Road, Chengdu, Sichuan 610072, P.R. China

E-mail: wenyingl@126.com

Key words: apoptosis, alveolar epithelial cells, hyperoxia, microRNA-16 and JAK/STAT3 signaling pathways. This may also represent a promising target for novel therapeutic strategies for acute lung injury.

\section{Introduction}

Acute lung injury (ALI) is a disease that is characterized by severe alveolar damage, such as alveolar spaces containing a protein-rich fluid and an acute inflammatory response, which lead to impaired pulmonary gas exchange, arterial hypoxemia, edema, and neutrophil and macrophage infiltration, and potentially respiratory failure $(1,2)$. The incidence of ALI is $10.4 \%$ and it is a common reason for the admission of patients to intensive care units worldwide $(3,4)$. At present, the identification and development of novel therapeutic strategies for ALI is urgently required. Although hyperoxia may be used to treat patients with acute and chronic cardiovascular and pulmonary diseases, a prolonged exposure to hyperoxia leads to severe ALI and impaired respiratory function $(5,6)$. In patients with hyperoxia, dysfunction of alveolar epithelial cells, including inhibited cell proliferation and enhanced cell apoptosis and necrosis $(7,8)$, has been reported. It has been hypothesized that hyperoxia-induced ALI may be associated with reactive oxygen species (ROS)-induced accumulation of inflammatory factors, such as transforming growth factor (TGF)- $\beta$, and direct insult (9).

microRNAs (miRNA/miRs) have emerged as an important class of gene expression regulators, some of which have been implicated in the dysregulation of key genes involved in ALI. It was previously demonstrated that miRNAs exert important functions in ALI (10) and may be novel biomarkers for predicting the mortality and treatment outcome of severe sepsis and sepsis-induced ALI (11). miR-181a significantly reduces lipopolysaccharide (LPS)-induced ALI in mice and A549 cells by targeting Bcl-2 (10). Furthermore, LPS-induced cell apoptosis and ALI in pulmonary microvascular endothelial cells is mediated by miR-1246 and its target gene, angiotensin-converting enzyme 2 (12). It was also demonstrated that miR-34a inhibits the excessive autophagic activity in alveolar type II epithelial cells (AECII) through targeting forkhead box O3, which subsequently reduces LPS-induced ALI (13). In addition, in A549 cells, it was reported that miR-16 modulates the expression of epithelial sodium channel protein and serotonin transporter in alveolar epithelial cells, 
and that the downregulation of TGF- $\beta$ by miR-16 in A549 cells has an important role in ALI $(14,15)$. However, the regulatory effect of miR-16 in cell apoptosis and ALI, and its underlying molecular mechanisms, remain unclear.

The present study aimed to investigate the regulatory role of miR-16 in phenotype, cell proliferation and apoptosis, and the potential involvement of TGF- $\beta /$ Smad family member $2(\mathrm{Smad} 2)$ and JAK/signal transducer and activator of transcription (STAT)3 signaling, in primary human AECII cells. TGF- $\beta /$ Smad 2 and JAK/STAT3 signaling pathways were investigated in the present study as they have been previously reported to exhibit important roles in AECII cells $(16,17)$. The results of the current study indicated that miR-16 may have an important role in the modulation of cell phenotype, proliferation and apoptosis in primary human AECII cells. In addition, miR-16 inhibited the TGF- $\beta / \mathrm{Smad} 2$ and JAK/STAT3 signaling pathways. Therefore, TGF- $\beta / \mathrm{Smad} 2$ and JAK/STAT3 signaling pathways may be implicated in hyperoxia-induced ALI and may be potential therapeutic targets.

\section{Materials and methods}

Cell culture. The human lung tissues (non-cancerous tissues) used for primary human AECII isolation were collected during resection of lung carcinoma, as previously described $(18,19)$. Written informed consent was obtained from all patients. The present study was approved by the Ethical Committee of the Hospital of the University of Electronic Science and Technology of China and Sichuan Provincial People's Hospital (Chengdu, China). Lung tissue sections were perfused with $0.15 \mathrm{M}$ sterile sodium chloride saline solution. The sections were then digested by trypsin $(0.25 \%)$ at $37^{\circ} \mathrm{C}$ for $45 \mathrm{~min}$. Then, the sections were incubated with $10 \%$ newborn calf serum (Invitrogen; Thermo Fisher Scientific, Inc., Waltham, MA, USA), shaken for $5 \mathrm{~min}$ with DNase $(250 \mu \mathrm{g} / \mathrm{ml})$ at $25^{\circ} \mathrm{C}$. Tissue debris was removed by $400 \mu \mathrm{m}$ and $40 \mu \mathrm{m}$ filters. Cells were then incubated at $37^{\circ} \mathrm{C}$ with $5 \% \mathrm{CO}_{2}$ for $2 \mathrm{~h}$ to remove the mononuclear cells and non-adherent ATII cells. Finally, AECII cells were maintained in DCCM-1 culture medium (Invitrogen; Thermo Fisher Scientific, Inc.) containing 10\% newborn calf serum (Invitrogen; Thermo Fisher Scientific, Inc.) and $1 \%$ penicillin-streptomycin-glutamine (Gibco; Thermo Fisher Scientific, Inc.) at $37^{\circ} \mathrm{C}$ with $5 \% \mathrm{CO}_{2}$. As previously described, AECII cells positively expressed surfactant protein (SP)-A and C, and maintained their phenotype for up to 6 days (20). In the present study, the expression of SP-A was detected by immunofluorescence.

Cell transfection. miR-16 mimics and negative control (NC) miRNA were purchased from Shanghai GenePharma Co., Ltd. (Shanghai, China). Cells $\left(2 \times 10^{5}\right.$ cells/well) were seeded in 6 -well plates. The sequences of miR-16 mimics/NC miRNA were as follows: miR-16 mimics, 5'-UAGCAGCACGUAAAU AUUGGCG-3'; and NC miRNA, 5'-UUCUCCGAACGUGUC ACGUTT-3'. miR-16 mimics and NC miRNA (50 nM) were transfected into cells using Lipofectamine ${ }^{\circledR} 2000$ (Invitrogen; Thermo Fisher Scientific, Inc.) at $70 \%$ confluency. After $4 \mathrm{~h}$ of transfection, the culture medium was replaced with DCCM-1 culture medium (Invitrogen; Thermo Fisher Scientific, Inc.) containing 10\% newborn calf serum (Invitrogen; Thermo
Fisher Scientific, Inc.) and $1 \%$ penicillin-streptomycin-glutamine (Gibco; Thermo Fisher Scientific, Inc.) at $37^{\circ} \mathrm{C}$ with $5 \% \mathrm{CO}_{2}$. After $24 \mathrm{~h}$, cells were treated with hyperoxia and the miR-16 mimic-induced overexpression of miR-16 was confirmed by reverse transcription-quantitative polymerase chain reaction (RT-qPCR). Cells were subsequently collected for further experiments.

Exposure to air or hyperoxia. AECII cells were seeded on coverslips and placed in a 6-well plate until 70-80\% confluence at $37^{\circ} \mathrm{C}$ with $5 \% \mathrm{CO}_{2}$. For hyperoxia, cells were moved into a modular chamber that was filled with a gas mixture of $60 \%$ oxygen and $5 \% \mathrm{CO}_{2}$ at $37^{\circ} \mathrm{C}$. The control cells were placed in the chamber filled with air containing $5 \% \mathrm{CO}_{2}$ at $37^{\circ} \mathrm{C}$. The incubation time was $24 \mathrm{~h}$. A Pigeon I oxygen measuring meter (Guangdong Pigeon Medical Apparatus Co., Ltd., Guangdong, China) was used to monitor the oxygen fraction continuously.

$R T-q P C R$. Total RNA was isolated by TRIzol reagent (Invitrogen; Thermo Fisher Scientific, Inc.) (21) and reverse transcribed into cDNA using the iScript cDNA Synthesis kit (Bio-Rad Laboratories, Inc., Hercules, CA, USA) at $16^{\circ} \mathrm{C}$ for $30 \mathrm{~min}, 42^{\circ} \mathrm{C}$ for $30 \mathrm{~min}$ and $85^{\circ} \mathrm{C}$ for $5 \mathrm{~min}$. Subsequently, qPCR was performed on a Bio-Rad CFX96 Real-Time PCR Detection System (Bio-Rad Laboratories, Inc.) using SYBR ${ }^{\circledR}$ Green Real-Time PCR Master Mixes (Thermo Fisher Scientific, Inc.) (22) according to the following thermocycling conditions: $95^{\circ} \mathrm{C}$ for $10 \mathrm{~min}$ followed by 40 cycles at $95^{\circ} \mathrm{C}$ for $15 \mathrm{sec}$ and $60^{\circ} \mathrm{C}$ for $60 \mathrm{sec}$, and a final extension at $65^{\circ} \mathrm{C}$ for $3 \mathrm{~min}$. The $2^{-\Delta \Delta \mathrm{Cq}}$ method was used to quantify the relative expression (23). All primers were purchased from Clontech Laboratories, Inc. (Mountainview, CA, USA). The following primers were used: hsa-miR-16-5p 5'-ACACTCCAGCTGGGTAGCAGCACG TAAATATT-3' (forward) and 5'-CTCAACTGGTGTCGT GGAGTCGGCAATTCAGTTGAGCGCCAA-3' (reve rse); U6 5'-CTCGCTTCGGCAGCACA-3' (forward) and 5'-AAC GCTTCACGAATTTGCGT-3' (reverse); STAT3, 5'-ATC ACGCCTTCTACAGACTGC-3' (forward) and 5'-CATCCT GGAGATTCTCTACCACT-3' (reverse); JAK, 5'-ATCCAC CCAACCATGTCTTCC-3' (forward) and 5'-ATTCCATGC CGATAGGCTCTG-3' (reverse); aquaporin 5, 5'-GCTCAC TGGGTTTTCTGGGTA-3' (forward) and 5'-TCCATGGTCT TCTTCCGCTC-3' (reverse); SP-A, 5'-ACAGCCACCATT TGGAAGAAC-3' (forward) and 5'-CTGGGTCATGAGAGT GTGGTC-3' (reverse); Smad2, 5'-CCATCTCCTACTACG AGCTGAA-3' (forward) and 5'-CACTGCTGCATTCCT GTTGAC-3' (reverse); TGF- $\beta$ 5'-TGGAAACCCACAACG AAATC-3' (forward) and 5'-AAGGCGAAAGCCCTCAAT-3' (reverse); GAPDH, 5'-TGTTCGTCATGGGTGTGAAC-3' (forward) and 5'-ATGGCATGGACTGTGGTCAT-3' (reverse). Experiments were performed in triplicate.

Immunofluorescence staining. After transfection of AECII cells with miR-16 mimics, cells were incubated for 15-18 h and were exposed to air or $60 \%$ oxygen for $24 \mathrm{~h}$. Subsequently, the cells were rinsed with ice-cold PBS and fixed with $1 \%$ methanol for $15 \mathrm{~min}$ at $25^{\circ} \mathrm{C}$. Cells were blocked with $1 \%$ bovine serum albumin (Invitrogen; Thermo Fisher Scientific, Inc.) for $10 \mathrm{~min}$ at room temperature, incubated with anti-SP-A (cat. no. sc-80621; 1:200; Santa Cruz Biotechnology, Inc., 
A

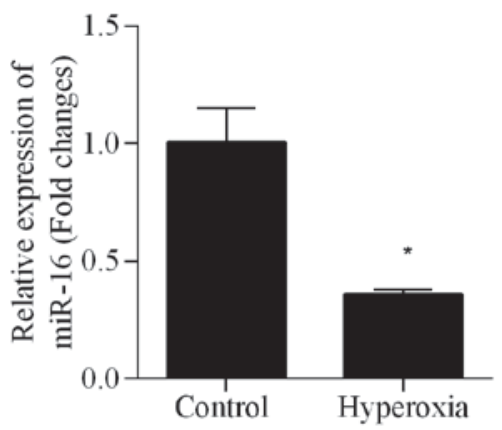

B

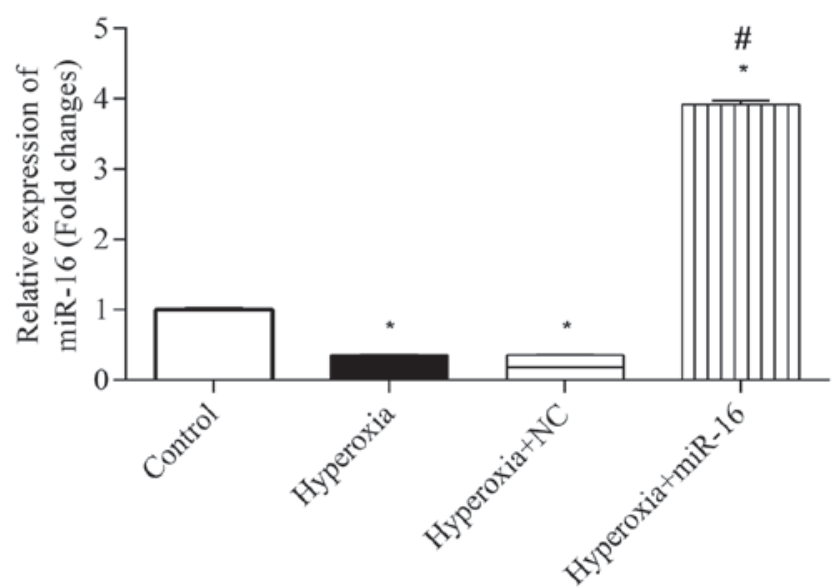

Figure 1. Expression levels of miR-16 in AECII cells following hyperoxia exposure. (A) Levels of miR-16 in AECII following hyperoxia exposure, as determined by RT-qPCR. (B) Levels of miR-16 in hyperoxia-exposed AECII cells following transfection with miR-16 mimics. "P $<0.05$ vs. control group; ${ }^{*} \mathrm{P}<0.05$ vs hyperoxia group and hyperoxia + NC groups. miR, microRNA; AECII, primary human alveolar type II epithelial cells; RT-qPCR, reverse transcription-quantitative polymerase chain reaction; NC, negative control.

Dallas, TX, USA) at $4^{\circ} \mathrm{C}$ overnight and then incubated with a fluorescein isothiocyanate (FITC)-conjugated secondary antibody (cat. no. sc-2364; 1:500; Santa Cruz Biotechnology, Inc.) for $1 \mathrm{~h}$ at room temperature. Nuclei were stained with DAPI (Invitrogen; Thermo Fisher Scientific, Inc.) at $25^{\circ} \mathrm{C}$ for $5 \mathrm{~min}$. Finally, the fluorescence was visualized using an Olympus IX70 fluorescence microscope (magnification, x40; Olympus Corporation, Tokyo, Japan).

Cell Counting Kit (CCK)-8 assay. Cell viability was measured quantitatively using a CCK-8 assay (Beyotime Institute of Biotechnology, Haimen, China), according to the manufacturer's protocol. Briefly, cell viability was detected in 96-well plates $\left(2 \times 10^{3}\right.$ cells per well), and absorbance was assessed using a microplate reader (Molecular Devices, LLC, Sunnyvale, CA, USA) at $450 \mathrm{~nm}$ following incubation with CCK- 8 solution for $30 \mathrm{~min}$.

Cell apoptosis assay. Cell apoptosis was performed using an FITC Apoptosis Detection Kit II (BD Biosciences, Franklin Lakes, NJ, USA), according to the manufacturer's protocol. Cells were harvested following treatment. After suspension in $100 \mu 1$ annexin binding buffer, cells $\left(1 \times 10^{5}\right)$ were incubated with $10 \mu 1$ annexin V-FITC (20 $\mu \mathrm{g} / \mathrm{ml})$ for $15 \mathrm{~min}$ on ice in the dark. Subsequently, $400 \mu 1$ PBS and $5 \mu 1$ PI $(50 \mu \mathrm{g} / \mathrm{ml})$ were added, and cell apoptosis was immediately detected by flow cytometry using the BD FACSCanto ${ }^{\mathrm{TM}}$ II system (BD Biosciences).

Western blotting. Proteins were extracted from AECII cells using radioimmunoprecipitation assay buffer (Beyotime Institute of Biotechnology) and the protein concentration was measured by a Bicinchoninic Acid assay kit (Pierce; Thermo Fisher Scientific, Inc.). Equal amounts of protein lysates $(40 \mu \mathrm{g})$ were separated by $15 \%$ SDS-PAGE and transferred to polyvinylidene difluoride membranes (EMD Millipore, Billerica, MA, USA). Membranes were blocked with $5 \%$ non-fat milk at $25^{\circ} \mathrm{C}$ for $30 \mathrm{~min}$, and then incubated with primary antibodies against TGF- $\beta$ (1:500; cat. no. ab92486; Abcam, Cambridge, MA,
USA), Smad2 (1:500; cat. no. ab40855; Abcam), phosphorylated (p)-Smad2 (1:500; cat. no. ab53100; Abcam), JAK (1:500; cat. no. ab39636; Abcam), STAT3 (1:200; cat. no. ab68153; Abcam) or GAPDH (1:1,000; cat. no. ab8245; Abcam) overnight at $4^{\circ} \mathrm{C}$, and subsequently incubated with the following secondary antibodies: Anti-Rabbit IgG VHH single domain antibody $(1: 1,000$; cat. no. ab191866; Abcam) or donkey anti-Rabbit IgG H\&L (DyLight $^{\circledR} 488$ ) antibody (1:100; cat. no. ab98488; Abcam) for $1 \mathrm{~h}$ at room temperature. Both secondary antibodies were conjugated with horseradish peroxidase. The blots were detected using Enhanced Chemiluminescence Plus reagent Pierce ${ }^{\mathrm{TM}}$ ECL Western Blotting Substrate (Thermo Fisher Scientific, Inc.), according to the manufacturer's protocol. Protein expression was quantified via densitometric analysis using Gel-pro analyzer 4.0 (Media Cybernetics, Inc., Rockville, MD, USA).

Statistical analysis. Data were analyzed using SPSS v17.0 (SPSS, Inc., Chicago, IL, USA) with paired t-tests (for two groups) or one-way analysis of variance followed by Tukey's post-hoc test (for three or more groups). All experiments were repeated at least three times and data are presented as mean \pm standard deviation. $\mathrm{P}<0.05$ was considered to indicate a statistically significant difference.

\section{Results}

Hyperoxia suppresses the expression of miR-16. Following hyperoxia exposure in mice, it was previously demonstrated that the lung expression of miR-16 was significantly reduced (15). To investigate the function of miR-16 in hyperoxia-induced ALI, the current study analyzed the expression of miR-16 in primary human AECII cells. The results demonstrated that miR-16 levels were significantly decreased in AECII cells following hyperoxia exposure (Fig. 1A), compared with control cells, indicating that the downregulation of miR-16 may be associated with hyperoxia-induced ALI. Following transfection of AECII cells with miR-16 mimics, the expression of miR-16 was significantly increased in hyperoxia-exposed AECII cells (Fig. 1B). Subsequently, further experiments were 
A

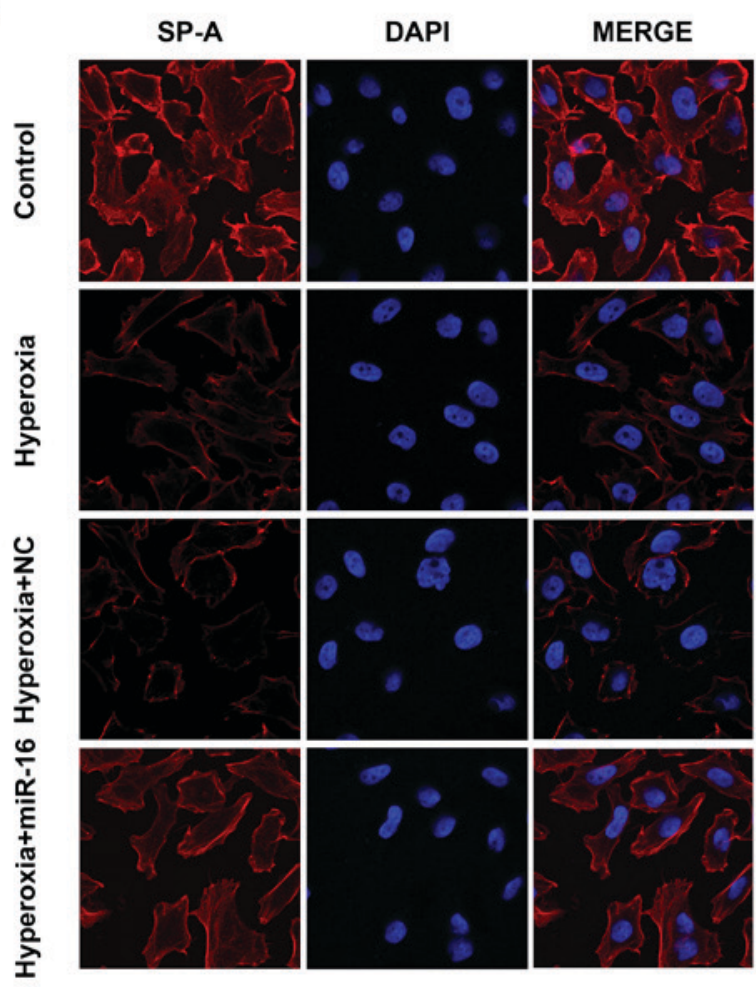

B

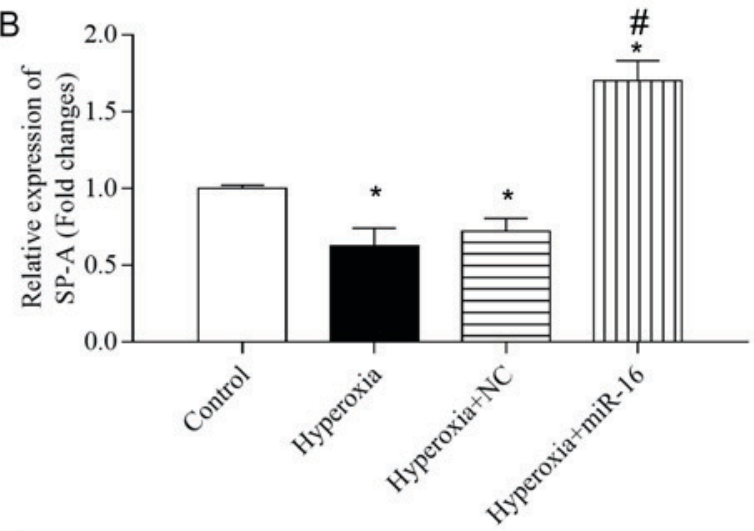

C

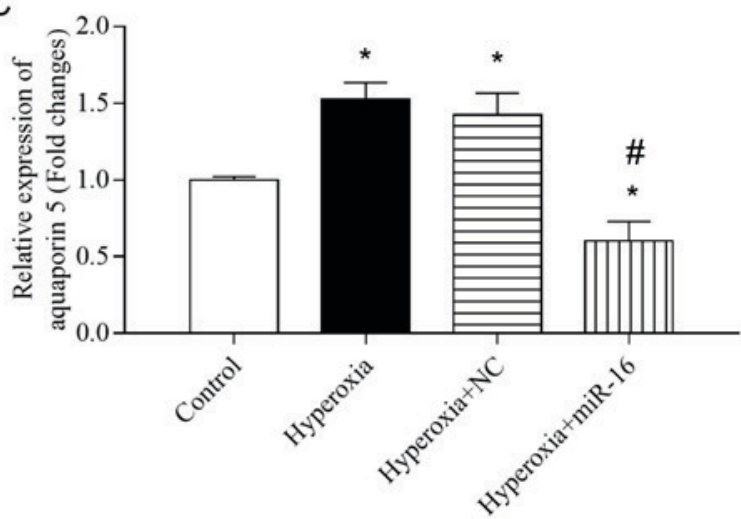

Figure 2. Levels of SP-A and aquaporin 5 in AECII cells following hyperoxia. Cells were transfected with or without miR-16 mimics and subsequently treated with hyperoxia for $24 \mathrm{~h}$. (A) Immunofluorescence of SP-A in AECII cells treated with hyperoxia (magnification, x40). Blue staining indicates DAPI staining of nuclei and red staining indicates SP-A immunostaining. The mRNA expression of (B) SP-A and (C) aquaporin 5 was determined by reverse transcription-quantitative polymerase chain reaction. ${ }^{*} \mathrm{P}<0.05$ vs. control group; ${ }^{~} \mathrm{P}<0.05$ vs. hyperoxia group and hyperoxia $+\mathrm{NC}$ groups. SP-A, surfactant protein-A; AECII, primary human alveolar type II epithelial cells; miR, microRNA; NC, negative control.

performed to determine the effect of miR-16 on phenotype, ALI, proliferation and cell apoptosis.

miR-16 rescues $S P$-A expression in hyperoxia-exposed AECII cells. SP-A is a biomarker of AECII cells as it is only secreted by AECII cells (24). The SP-A expression in AECII cells was detected using immunofluorescence (Fig. 2A). The results demonstrated that the expression of SP-A in the cytoplasm was markedly reduced by hyperoxia, compared with the control cells. However, transfection of AECII cells with miR-16 mimics rescued the SP-A fluorescence in hyperoxia-treated cells (Fig. 2A). Consistently, the expression of SP-A mRNA was significantly inhibited by hyperoxia, compared with control cells, and miR-16 mimics enhanced the SP-A mRNA expression in hyperoxia-exposed AECII cells (Fig. 2B).

Furthermore, the mRNA expression of aquaporin 5 was determined by RT-qPCR. The expression of aquaporin 5 was upregulated by hyperoxia, compared with control cells, and this effect was suppressed by miR-16 mimic transfection in hyperoxia-exposed AECII cells (Fig. 2C). This indicates that transdifferentiation disorder from AECII to alveolar type I epithelial cells was induced by hyperoxia, and this effect was inhibited by miR-16. Therefore, miR-16 may have a protective effect in AECII cells against hyperoxia-induced ALI.

miR-16 reverses the hyperoxia-induced suppression of cell proliferation and induction of cell apoptosis. It has been previously reported that hyperoxia induced AECII apoptosis (25). In order to investigate the effect of hyperoxia on AECII cells, cell proliferation was detected by a CCK-8 assay and apoptosis was determined by flow cytometry (Fig. 3). The cell proliferation was suppressed by hyperoxia at 48, 72 and 96 h, compared with the control group (Fig. 3A). The role of miR-16 in cell proliferation in hyperoxia was also investigated by overexpressing miR-16 in AECII cells; the results demonstrated that the cell proliferation was significantly increased by transfection with miR-16 mimics in hyperoxia-exposed AECII cells, compared with the hyperoxia + NC group and hyperoxia $+\mathrm{NC}$ groups. In addition, the percentage of apoptotic cells was increased following hyperoxia exposure, compared with the control group (Fig. 3B). The effect of miR-16 on cell apoptosis was also investigated using miR-16 mimics, which indicated that cell apoptosis was significantly inhibited by the miR-16 mimics in hyperoxia, compared with the NC group and hyperoxia $+\mathrm{NC}$ groups. These results indicate that hyperoxia triggered apoptosis and suppressed cell proliferation in AECII cells, which was reversed by transfection with miR-16 mimics.

miR-16 alters TGF- $\beta /$ Smad2 and JAK/STAT3 pathways in AECII cells in vitro. By using A549 cells, it was previously reported that miR-16 modulates the expression of epithelial sodium channel protein and serotonin transporter in alveolar epithelial cells, and that miR-16 downregulated TGF- $\beta$ in A549 cells has a critical role in ALI (15). The 

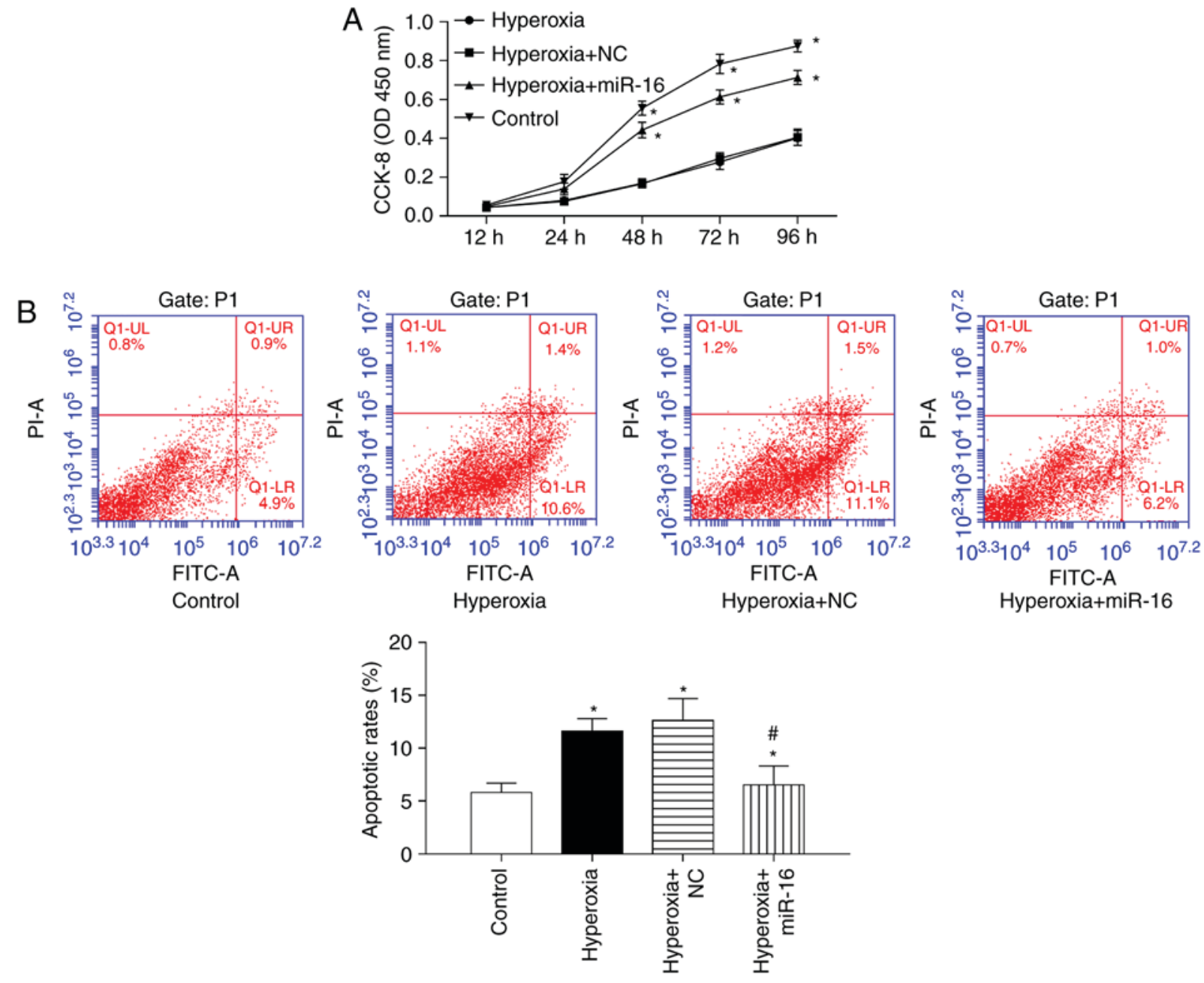

Figure 3. Effects of miR-16 mimics on hyperoxia-induced inhibition of cell proliferation and promotion of cell apoptosis in AECII cells. Following transfection with miR-16 mimics or NC, cells were exposed to hyperoxia for $24 \mathrm{~h}$. (A) Cell proliferation was determined by a CCK-8 assay. "P $<0.05$ vs. hyperoxia group and hyperoxia + NC groups. (B) Apoptosis was determined by annexin V-FITC/PI staining using flow cytometry. LR and UR quadrants were considered to indicate apoptotic cells. ${ }^{\mathrm{P}}<0.05$ vs. control group; ${ }^{\prime} \mathrm{P}<0.05$ vs. hyperoxia group and hyperoxia $+\mathrm{NC}$ groups. miR, microRNA; AECII, primary human alveolar type II epithelial cells; NC, negative control; CCK-8, Cell Counting Kit-8; FITC, fluorescein isothiocyanate; PI, propidium iodide; OD, optical density.

present study therefore investigated TGF- $\beta / \mathrm{Smad} 2$ and JAK/STAT3 pathways in AECII cells. To determine the role of miR-16 in modulating the TGF- $\beta /$ Smad 2 and JAK/STAT3 pathways, the mRNA and/or protein expression of TGF- $\beta$, Smad2, p-Smad2, JAK and STAT3 was detected in miR-16 mimic-transfected AECII cells following hyperoxia (Fig. 4). The results demonstrated that hyperoxia significantly induced the mRNA expression of TGF- $\beta$ (Fig. 4A), Smad2 (Fig. 4B), JAK (Fig. 4C) and STAT3 (Fig. 4D). However, transfection of miR-16 mimics significantly inhibited the hyperoxia-induced mRNA expression of TGF- $\beta$ (Fig. 4A), Smad2 (Fig. 4B), JAK (Fig. 4C) and STAT3 (Fig. 4D).

Consistent with these results, increased protein levels of TGF- $\beta$, Smad2, p-Smad2, JAK and STAT3 were observed in AECII cells following hyperoxia (Fig. 4E). However, there was a significant reduction in the protein expression of TGF- $\beta$, Smad2, p-Smad2, JAK and STAT3 in miR-16 mimic-transfected AECII cells following hyperoxia, compared with the NC group. Therefore, miR-16 is associated with reduced expression of TGF- $\beta$, Smad2, p-Smad2, JAK and STAT3, indicating that miR-16 may regulate hyperoxia-induced ALI through TGF- $\beta /$ Smad2 and JAK/STAT3 signaling pathways.

\section{Discussion}

The results of the present study demonstrated that miR-16 was downregulated in primary human AECII cells in ALI, which was associated with the TGF- $\beta / \mathrm{Smad} 2$ and JAK/STAT3 pathways.

In A549 human non-small cell lung carcinoma cells, overexpression of miR-16 by miR-16 mimics significantly suppressed cell proliferation and significantly promoted cell apoptosis (26). Furthermore, overexpression of miR-16 significantly increased the levels of p27, Bcl-2-associated X, procaspase 3 and cleaved caspase 3 , and decreased the levels of Bcl-2 (26). In the current study, the results demonstrated that miR-16 was downregulated following hyperoxia exposure, which indicated that downregulation of miR-16 may be associated with hyperoxia-induced ALI. The cell proliferation was suppressed by hyperoxia; however, cell proliferation was increased in the miR-16 mimic transfection group compared with the NC group following hyperoxia. In addition, the percentage of apoptotic cells was significantly increased following hyperoxia exposure, compared with the control group, and the cell apoptosis was reduced in the miR-16 mimic 
A
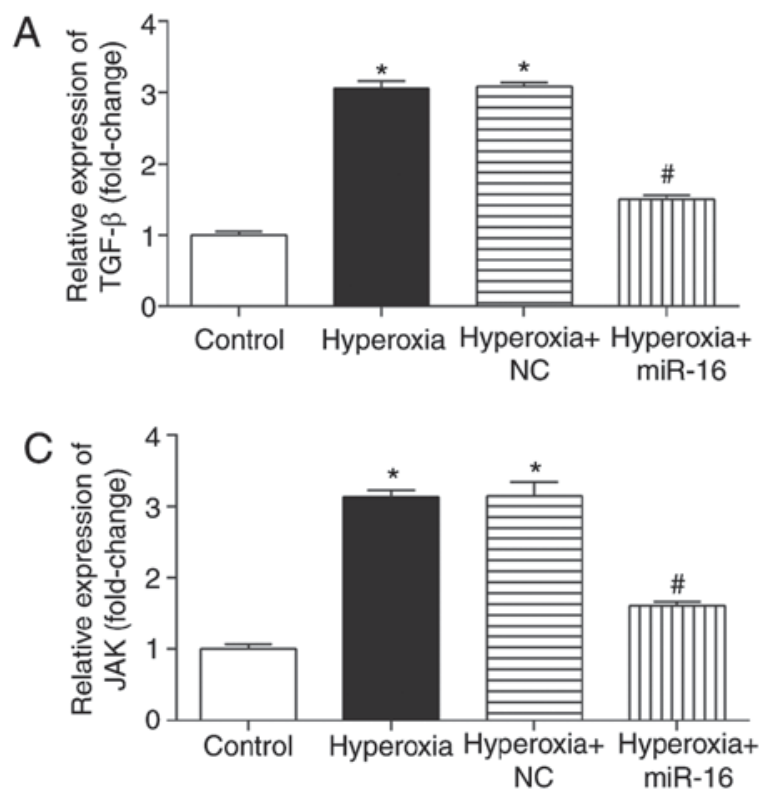

E

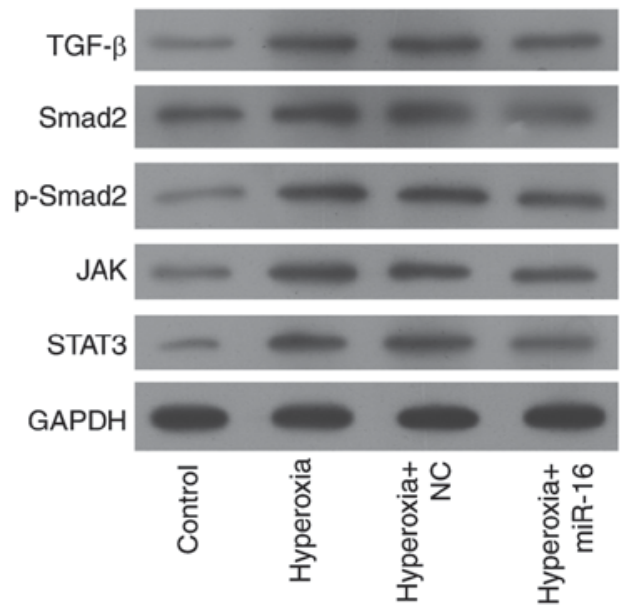

$\mathrm{B}$
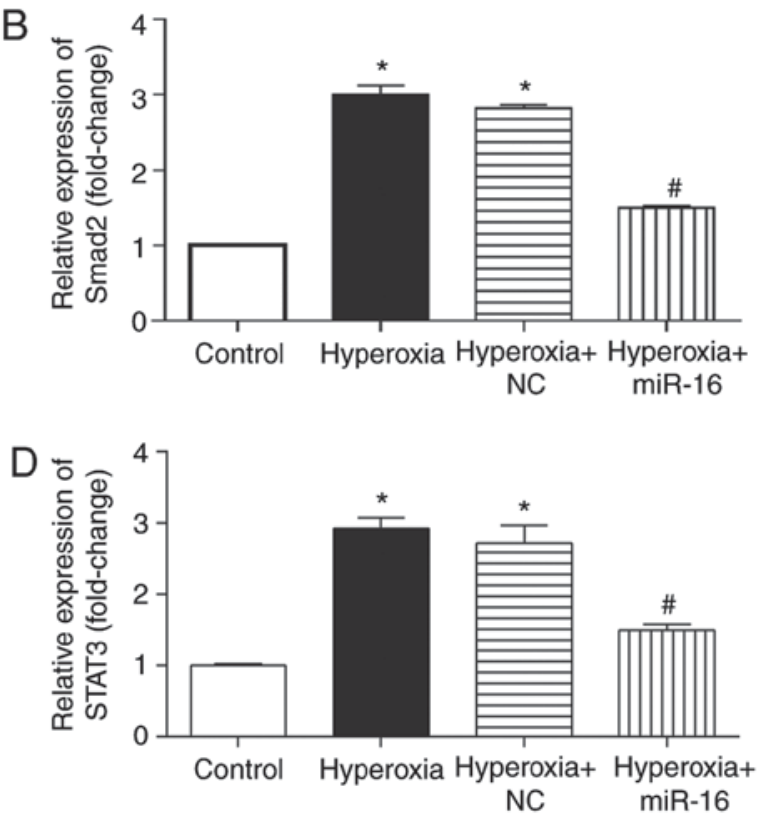

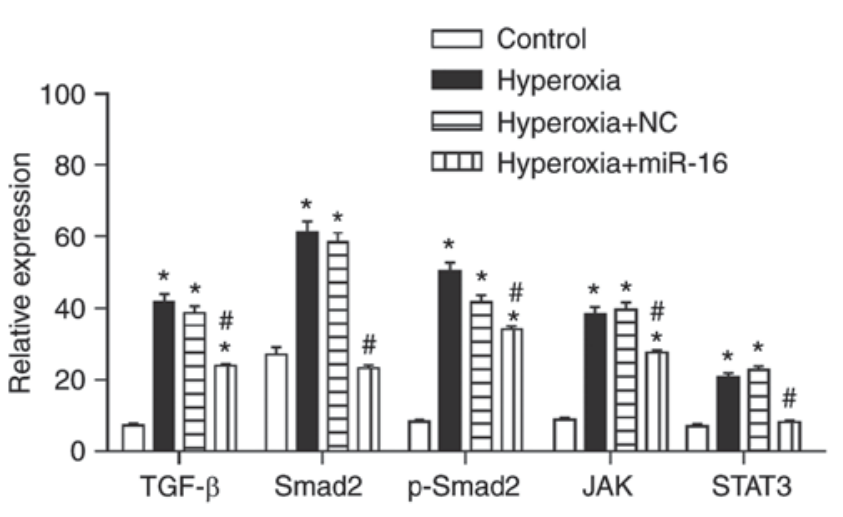

Figure 4. Transfection with miR-16 mimics modulates TGF- $\beta$ /Smad2 and JAK/STAT3 pathways in AECII cells. Following transfection with miR-16 mimics or NC, cells were exposed to hyperoxia for $24 \mathrm{~h}$. Subsequently, the mRNA expression of (A) TGF- $\beta$, (B) Smad2, (C) JAK and (D) STAT3 was determined by reverse transcription-quantitative polymerase chain reaction. (E) Protein expression of TGF- $\beta$, Smad2, p-Smad2, JAK and STAT3 was also detected in AECII cells by western blot analysis. Representative western blot bands are presented. " $\mathrm{P}<0.05$ vs. control group; ${ }^{*} \mathrm{P}<0.05$ vs. hyperoxia group and hyperoxia $+\mathrm{NC}$ groups. miR, microRNA; TGF $\beta$, transforming growth factor $\beta ;$, Smad2, Smad family member 2 ; STAT3, signal transducer and activator of transcription 3; AECII, primary human alveolar type II epithelial cells; NC, negative control; p-, phosphorylated-

transfection group compared with the NC group following hyperoxia. Therefore, hyperoxia triggered apoptosis and suppressed cell proliferation, which was reversed by transfection with miR-16 mimics in AECII cells.

In A549 cells, it was reported that miR-16 modulated the expression of epithelial sodium channel protein and serotonin transporter in alveolar epithelial cells, and that miR-16 downregulated TGF- $\beta$ in those cells (14). It was also reported that Smad2 knockout mice exhibited embryonic lethality, which indicated that TGF- $\beta$-mediated Smad 2 signaling may contribute to the development process $(22,24)$. Furthermore, in Smad3 knockout mice, weakened alveoli and emphysema were observed later in life $(22,24)$. Exogenous and endogenous TGF- $\beta$ may exhibit similar or different roles in diseases (27). In homeostasis of AECII cells in vitro, the addition of exogenous TGF- $\beta$ in culture medium protected AECII cells that were isolated from hyperoxic animals by suppressing hyperoxia-induced damage of DNA and repairing DNA, enhancing the secretion of fibronectin and vascular endothelial growth factor, and accelerating cell migration and would healing via Smad signaling (28), which indicates an important role for TGF- $\beta$ in hyperoxic animals. The present study therefore investigated the TGF- $\beta / \mathrm{Smad} 2$ pathway in AECII cells. The results demonstrated that hyperoxia significantly induced the expression of TGF- $\beta, \operatorname{Smad} 2$ and $\mathrm{p}-\operatorname{Smad} 2$, compared with the control group. In addition, transfection of AECII cells with miR-16 mimics markedly inhibited the hyperoxia-induced levels of TGF- $\beta, \operatorname{Smad} 2$ and $\mathrm{p}-\mathrm{Smad} 2$. It was previously reported that hyperoxia induced increases in the levels of active Smad 2 and TGF- $\beta$ (28). This is consistent with the results of the current study, which indicated that hyperoxia induced active Smad 2 and TGF- $\beta$ levels in AECII. The results also demonstrated that miR-16 mimic transfection downregulated the levels of TGF- $\beta$ and active Smad2, therefore protecting 
AECII cells. However, Buckley et al (28) demonstrated that exogenous TGF- $\beta$ protected the AECII cells via activation of Smad2. We hypothesize that these conflicting observations, whether the activation or inhibition of Smad2 leads to protective effects against hyperoxia, may be due to the source of cells. For example, Buckley et al (28) isolated the adult AECII cells from lavaged lungs, while the present study isolated adult AECII cells from human lung tissues (non-cancerous tissues) that were collected during resection of lung carcinoma. The results of the current study demonstrate an association between miR-16 expression and reduced expression of TGF- $\beta, \operatorname{Smad} 2$ and $\mathrm{p}-\mathrm{Smad} 2$, indicating that the TGF- $\beta /$ Smad 2 pathway may be regulated by miR-16 and be involved in hyperoxia-induced ALI. The present study focused on the role of miR-16, and the effect of TGF- $\beta$ expression should be investigated in future studies to determine whether underexpression or overexpression of TGF- $\beta$ protects AECII cells.

It was previously demonstrated that the JAK/STAT signaling pathway alters cell function via ROS, which has an important role in ALI $(29,30)$. Suppression of LPS-induced STAT3 activation was reported to protect cells against LPS-induced ALI (29). The present study also investigated the JAK/STAT3 pathway in AECII cells. The results demonstrated that hyperoxia significantly induced the expression of JAK and STAT3, while transfection of miR-16 significantly inhibited the hyperoxia-induced mRNA expression of JAK and STAT3 in AECII cells, indicating that miR-16 may protect against hyperoxia-induced ALI through JAK/STAT3 pathways. However, the target gene by which miR-16 directly controls the TGF- $\beta /$ Smad 2 and JAK/STAT3 pathways remains unclear.

In conclusion, the results of the current study demonstrated that overexpression of miR-16 exerted a protective effect in AECII cells against cell apoptosis and ALI, which may be associated with TGF- $\beta /$ Smad 2 and JAK/STAT3 pathways. Therefore, TGF- $\beta /$ Smad 2 and JAK/STAT3 pathways may be important novel therapeutic targets for hyperoxia-induced ALI.

\section{Acknowledgements}

The present study was supported by the National Natural Science Foundation of China (grant no. 81302161) and the Youth Science and Technology Foundation of Sichuan Provincial People's Hospital (grant no. 30305030858).

\section{Competing interests}

The authors declare that they have no competing interests.

\section{References}

1. Pittet JF, Griffiths MJ, Geiser T, Kaminski N, Dalton SL, Huang X, Brown LA, Gotwals PJ, Koteliansky VE, Matthay MA and Sheppard D: TGF-beta is a critical mediator of acute lung injury. J Clin Invest 107: 1537-1544, 2001.

2. Kallet RH and Matthay MA: Hyperoxic acute lung injury. Respir Care 58: 123-141, 2013.

3. Bellani G, Laffey JG, Pham T, Fan E, Brochard L, Esteban A Gattinoni L, van Haren F, Larsson A, McAuley DF, et al: Epidemiology, patterns of care, and mortality for patients with acute respiratory distress syndrome in intensive care units in 50 countries. JAMA 315: 788-800, 2016.
4. Fu H, Zhang T, Huang R, Yang Z, Liu C, Li M, Fang F and Xu F: Calcitonin gene-related peptide protects type II alveolar epithelial cells from hyperoxia-induced DNA damage and cell death. Exp Ther Med 13: 1279-1284, 2017.

5. Ward NS, Waxman AB, Homer RJ, Mantell LL, Einarsson O, $\mathrm{Du} \mathrm{Y}$ and Elias JA: Interleukin-6-induced protection in hyperoxic acute lung injury. Am J Respir Cell Mol Biol 22: 535-542, 2000.

6. Fukumoto J, Fukumoto I, Parthasarathy PT, Cox R, Huynh B, Ramanathan GK, Venugopal RB, Allen-Gipson DS, Lockey RF and Kolliputi N: NLRP3 deletion protects from hyperoxia-induced acute lung injury. Am J Physiol Cell Physiol 305: C182-C189, 2013.

7. Durr RA, Dubaybo BA and Thet LA: Repair of chronic hyperoxic lung injury: Changes in lung ultrastructure and matrix. Exp Mol Pathol 47: 219-240, 1987.

8. Wang Y, Feinstein SI, Manevich Y, Ho YS and Fisher AB: Lung injury and mortality with hyperoxia are increased in peroxiredoxin 6 gene-targeted mice. Free Radic Biol Med 37: 1736-1743, 2004.

9. Warner BB, Stuart LA, Papes RA and Wispé JR: Functional and pathological effects of prolonged hyperoxia in neonatal mice. Am J Physiol 275: L110-L117, 1998.

10. Li W, Qiu X, Jiang H, Han Y, Wei D and Liu J: Downregulation of miR-181a protects mice from LPS-induced acute lung injury by targeting Bcl-2. Biomed Pharmacother 84: 1375-1382, 2016.

11. Han Y, Li Y and Jiang Y: The prognostic value of plasma MicroRNA-155 and MicroRNA-146a level in severe sepsis and sepsis-induced acute lung injury patients. Clin Lab 62: 2355-2360, 2016

12. Fang Y, Gao F, Hao J and Liu Z: microRNA-1246 mediates lipopolysaccharide-induced pulmonary endothelial cell apoptosis and acute lung injury by targeting angiotensin-converting enzyme 2. Am J Transl Res 9: 1287-1296, 2017.

13. Song L, Zhou F, Cheng L, Hu M, He Y, Zhang B, Liao D and Xu Z: MicroRNA-34a suppresses autophagy in alveolar type II epithelial cells in acute lung injury by inhibiting FoxO3 expression. Inflammation 40: 927-936, 2017.

14. Frank J, Roux J, Kawakatsu H, Su G, Dagenais A, Berthiaume Y, Howard M, Canessa CM, Fang X, Sheppard D, et al: Transforming grow th factor-betal decreases expression of the epithelial sodium channel alphaENaC and alveolar epithelial vectorial sodium and fluid transport via an ERK1/2-dependent mechanism. J Biol Chem 278: 43939-43950, 2003.

15. Tamarapu Parthasarathy P, Galam L, Huynh B, Yunus A, Abuelenen T, Castillo A, Kollongod Ramanathan G, Cox R Jr and Kolliputi N: MicroRNA 16 modulates epithelial sodium channel in human alveolar epithelial cells. Biochem Biophys Res Commun 426: 203-208, 2012.

16. Zhou Y, He Z, Gao Y, Zheng R, Zhang X, Zhao L and Tan M: Induced pluripotent stem cells inhibit bleomycin-induced pulmonary fibrosis in mice through suppressing TGF- $\beta 1 /$ Smad-mediated epithelial to mesenchymal transition. Front Pharmacol 7: 430, 2016.

17. Dong Z, Tai W, Lei W, Wang Y, Li Z and Zhang T: IL-27 inhibits the TGF- $\beta 1$-induced epithelial-mesenchymal transition in alveolar epithelial cells. BMC Cell Biol 17: 7, 2016.

18. Sweeney S, Theodorou IG, Zambianchi M, Chen S, Gow A, Schwander S, Zhang JJ, Chung KF, Shaffer MS, Ryan MP, et al: Silver nanowire interactions with primary human alveolar type-II epithelial cell secretions: Contrasting bioreactivity with human alveolar type-I and type-II epithelial cells. Nanoscale 7: 10398-10409, 2015.

19. Witherden IR and Tetley TD: Isolation and culture of human alveolar type II pneumocytes. Methods Mol Med 56: 137-146, 2001.

20. Witherden IR, Vanden Bon EJ, Goldstraw P, Ratcliffe C, Pastorino U and Tetley TD: Primary human alveolar type II epithelial cell chemokine release: Effects of cigarette smoke and neutrophil elastase. Am J Respir Cell Mol Biol 30: 500-509, 2004.

21. Yan W, Wu Q, Yao W, Li Y, Liu Y, Yuan J, Han R, Yang J, Ji X and Ni C: MiR-503 modulates epithelial-mesenchymal transition in silica-induced pulmonary fibrosis by targeting PI3K p 85 and is sponged by lncRNA MALAT1. Sci Rep 7: 11313, 2017.

22. Liu JX, Yan ZP, Zhang YY, Wu J, Liu XH and Zeng Y: Hemodynamic shear stress regulates the transcriptional expression of heparan sulfate proteoglycans in human umbilical vein endothelial cell. Cell Mol Biol (Noisy-le-Grand) 62: 28-34, 2016

23. Livak KJ and Schmittgen TD: Analysis of relative gene expression data using real-time quantitative PCR and the 2(-Delta Delta C(T)) method. Methods 25: 402-408, 2001. 
24. Li F, He J, Wei J, Cho WC and Liu X: Diversity of epithelial stem cell types in adult lung. Stem Cells Int 2015: 728307, 2015.

25. De Paepe ME, Mao Q, Chao Y, Powell JL, Rubin LP and Sharma S: Hyperoxia-induced apoptosis and Fas/FasL expression in lung epithelial cells. Am J Physiol Lung Cell Mol Physiol 289: L647-L659, 2005.

26. Wang W, Chen J, Dai J, Zhang B, Wang F and Sun Y: MicroRNA-16-1 inhibits tumor cell proliferation and induces apoptosis in A549 Non-small cell lung carcinoma cells. Oncol Res 24: 345-351, 2016.

27. Zeng Y, Yao X, Chen L, Yan Z, Liu J, Zhang Y, Feng T, Wu J and Liu X: Sphingosine-1-phosphate induced epithelial-mesenchymal transition of hepatocellular carcinoma via an MMP-7/syndecan-1/TGF- $\beta$ autocrine loop. Oncotarget 7: 63324-63337, 2016.
28. Buckley S, Shi W, Barsky L and Warburton D: TGF-beta signaling promotes survival and repair in rat alveolar epithelial type 2 cells during recovery after hyperoxic injury. Am J Physiol Lung Cell Mol Physiol 294: L739-L748, 2008.

29. Zhao J, Yu H, Liu Y, Gibson SA, Yan Z, Xu X, Gaggar A, Li PK, Li C, Wei S, et al: Protective effect of suppressing STAT3 activity in LPS-induced acute lung injury. Am J Physiol Lung Cell Mol Physiol 311: L868-L880, 2016.

30. Byfield G, Budd S and Hartnett ME: The role of supplemental oxygen and JAK/STAT signaling in intravitreous neovascularization in a ROP rat model. Invest Ophthalmol Vis Sci 50: 3360-3365, 2009. 